\title{
Propofol postconditioning alleviates diabetic myocardial ischemia-reperfusion injury via the miR-200c-3p/AdipoR2/STAT3 signaling pathway
}

\author{
LIJUN HUANG, LI DING, SHENGHUI YU, XIN HUANG and QIUSHENG REN
}

Department of Anesthesiology, The Affiliated People's Hospital of Ningbo University, Ningbo, Zhejiang 315040, P.R. China

Received January 28, 2021; Accepted August 20, 2021

DOI: $10.3892 / \mathrm{mmr} .2022 .12653$

\begin{abstract}
Myocardial ischemia/reperfusion (MI/RI) syndrome is one of the leading causes of mortality and disability. Propofol postconditioning is known to improve myocardial ischemia/reperfusion injury (MI/RI). The present study aimed to explore the mechanism of propofol postconditioning in diabetic MI/RI. Diabetic MI/RI rat models were established and the rats were treated via propofol postconditioning. Staining with 2,3,5-triphenyl-2H-tetrazolium chloride, H\&E staining, TUNEL staining and ELISA were applied to detect infarct size, pathological changes, apoptosis and oxidative stress-related factor and apoptotic factor levels, respectively. Subsequently, the effect of propofol on H9C 2 cells was also assessed using the Cell Counting Kit- 8 assay. High-glucose hypoxia/reperfusion (H/R) models of H9C2 cardiomyocytes were established. miR-200c-3p overexpression or AdipoR2 silencing combined with propofol postconditioning was performed in $\mathrm{H} / \mathrm{R}$-induced $\mathrm{H} 9 \mathrm{C} 2$ cells and STAT3 protein expression levels were determined. Propofol postconditioning significantly reduced myocardial infarct size, oxidative stress and apoptosis in diabetic MI/RI models. Furthermore, propofol postconditioning significantly reduced the oxidative stress and apoptosis of $\mathrm{H} 9 \mathrm{C} 2$ cells in high-glucose H/R models. Propofol postconditioning also significantly downregulated miR-200c-3p expression levels and promoted AdipoR 2 expression levels. miR-200c-3p overexpression or AdipoR2 downregulation significantly reversed the effects of propofol postconditioning on its antioxidation and anti-apoptotic effects in $\mathrm{H} 9 \mathrm{C} 2$ cells and on decreasing STAT3 phosphorylation levels. Together, the results of the present study demonstrated that propofol postconditioning inhibited miR-200c-3p, upregulated AdipoR2 and activated
\end{abstract}

Correspondence to: Mrs.Lijun Huang, Department of Anesthesiology, The Affiliated People's Hospital of Ningbo University, 251 East Baizhang Road, Ningbo, Zhejiang 315040, P.R. China

E-mail: huanglijunyzz@163.com

Key words: diabetic myocardial ischemia-reperfusion, propofol postconditioning, microRNA-200c-3p, adiponectin receptor 2, STAT3, oxidative stress, apoptosis, cardiomyocyte the STAT3 signaling pathway, thus alleviating diabetic $\mathrm{MI} / \mathrm{RI}$ and therefore highlighting its potential as a treatment of diabetic MI/RI.

\section{Introduction}

Diabetes, a widespread metabolic disorder, primarily manifests as chronic hyperglycemia and is caused by insulin dysfunction or impaired insulin secretion, or a combination of both (1). Patients with diabetes are further predisposed to a greater risk of acquiring ischemic heart disease and myocardial ischemia-reperfusion injury (MI/RI) (2). In lieu of the increasing incidence rate of diabetes complicated with myocardial ischemia, it is important to explore new avenues to relieve MI/RI in diabetic patients $(3,4)$. Currently, myocardial ischemia caused by the blockage of heart blood vessels is treated via reperfusion, restoring blood flow, however, the process of reperfusion is known to lead to ischemic myocardial injury (2). MI/RI contributes to the morbidity and mortality of a large range of pathologies, including ischemic stroke, myocardial infarction, acute kidney damage and trauma (5). Therefore, the present study aimed to investigate the cellular mechanisms between diabetic MI/RI and cardioprotective mechanisms.

Propofol, a widely-used intravenous anesthetic, is known to interact with numerous pathophysiological processes in different types of cancer (6). Furthermore, propofol can reduce myocardial cell apoptosis and thus exhibits a cardio-protective effect (7). Similarly, propofol is effective in alleviating hemodynamic instability in diabetic patients treated with glibenclamide (8). As a result of its anti-oxidant properties, propofol protects against MI/RI (9). It has also been shown that propofol may protect against MI/RI by reducing oxidative stress and inhibiting cardiomyocyte apoptosis (10). Nevertheless, the role of propofol in diabetic MI/RI remains unclear.

Propofol has previously been reported to protect against hepatic I/R via upregulation of microRNAs (miRNAs) (11). Inherently, miRNAs are small endogenous RNAs, which possess the ability to post-transcriptionally regulate gene expression and are regarded as crucial promising biomarkers for disease development (12). miRNAs have also been associated with diabetic MI/RI (2). For example, propofol is suggested to serve as a therapeutic target for MI/RI via interactions with 
miRNA (13). One of the most important regulatory factors in diabetic MI/RI is the interacting miRNA family. For example, miR-144, miR-34a, miR-200 and miR-155 are identified as being pro-apoptotic, among which miR-200c may be related to $\mathrm{MI} / \mathrm{RI}$ in diabetes (14). Moreover, inhibition of miR-200c is shown to diminish reactive oxygen species (ROS) production, lipid peroxidation and malondialdehyde (MDA) peroxidation and upregulate anti-oxidant enzyme superoxide dismutase (SOD) activity in cardiomyocytes (15). However, at present there is no evidence on whether propofol postconditioning can attenuate diabetic MI/RI through miR-200c-3p. Therefore, the present study aimed to investigate the protective mechanism of propofol postconditioning on diabetic MI/RI, hoping to provide a new theoretical basis for propofol postconditioning to attenuate diabetic MI/RI.

\section{Materials and methods}

Ethics statement. The present study was approved by the Academic Ethics Committee of The Affiliated People's Hospital of Ningbo University (approval number: 2019-010; Ningbo, China). Animal experimentation was carried out in strict accordance with the Guidelines for the Management and Use of Laboratory Animals issued by the Laboratory Association of China. Extensive efforts were made to minimize the suffering of the animals.

Diabetic MI/RI model establishment and grouping. A total of 54 healthy male Sprague-Dawley (SD) rats (age, 8-10 weeks; weight, 250-300 g) were purchased from Beijing Vital River Laboratory Animal Technology Co., Ltd. The rats were housed at $22-24^{\circ} \mathrm{C}$ with 12 -h light/dark cycles, at 50-60\% humidity and had free access to food and water.

The obtained SD rats were randomly assigned into a high-glucose (HG) group (fed with high fat and HG feed; $\mathrm{n}=54$ ). After four weeks all rats were fasted for $12 \mathrm{~h}$ and the rats were intraperitoneally injected with $65 \mathrm{mg} / \mathrm{kg}$ streptozotocin (Sigma-Aldrich; Merck KGaA) (16). Blood samples were collected from the tail vein after $72 \mathrm{~h}$ to assess fasting blood glucose levels. Diabetic rat models were regarded as successfully established when blood glucose levels were $\geq 16.7 \mathrm{mmol} / \mathrm{l}$ and the animals presented with diabetic symptoms, including excessive drinking, eating, polyuria and weight loss. Subsequently, the diabetic rats were fed with high-fat and HG feed and had their blood glucose monitored for 4 weeks. The rats with unqualified blood glucose were excluded and the qualified ones were used for I/R experiments.

Diabetic rats were intraperitoneally anesthetized with $2 \%$ pentobarbital sodium $(50 \mathrm{mg} / \mathrm{kg}$; Sigma-Aldrich; Merck KGaA). Following tracheal intubation, mechanical ventilation was performed with a ventilator. The ventilator was set to the following conditions: tidal volume, $6 \mathrm{ml} / \mathrm{kg}$; respiratory ratio, 1:1.5; and respiratory rate, 80 times $/ \mathrm{min}$. An 8-channel physiological signal recorder (PowerLab/8s; ADInstruments Pty Ltd.) was used to continuously monitor a 3-lead electrocardiogram (ECG). The femoral vein was then punctured, catheterized and connected with a micro-perfusion pump for infusion of propofol or normal saline. The left anterior descending coronary artery was isolated through thoracotomy. A 6/0 thread was threaded under the artery and a rigid polyethylene tube with a diameter of $2 \mathrm{~mm}$ and a length of $0.5 \mathrm{~cm}$ was placed between the myocardium and the ligation thread. When the ligation thread was tightened the color of the myocardium at the ligation site became dark and the ECG displayed significant elevation of the ST segment, indicating successful ischemia. After $30 \mathrm{~min}$ of ischemia the ligation thread was released, the color of heart turned back to red, the ST elevation dropped, and reperfusion was performed for $120 \mathrm{~min}$ to establish the MI/RI rat model (17).

Diabetic rats were randomly assigned into the following: the sham group (DS group), the MI/RI group (DI group) and the MI/RI + propofol group (DP group), with 18 rats in each group, and with 6 rats used for TTC staining, 6 rats for homogenization treatment, and 6 rats for section staining. Only chest opening and threading were performed in DS rats, equal volumes of normal saline were infused in DI rats and other operations were the same as those in the DP group. Propofol was infused at constant velocity into the femoral vein $3 \mathrm{~min}$ prior to perfusion in the DP group for $5 \mathrm{~min}$ (propofol was infused $3 \mathrm{~min}$ before the start of perfusion until $2 \mathrm{~min}$ after the start of perfusion) $(20 \mathrm{mg} / \mathrm{kg} / \mathrm{h})(18)$. Following I/R treatment, rats were euthanized by intraperitoneal injection of $2 \%$ pentobarbital sodium $(200 \mathrm{mg} / \mathrm{kg})$.

\section{2,3,5-triphenyl-2H-tetrazolium chloride (TTC) staining. The} hearts of rats in each group were removed and sliced into $2 \mathrm{~mm}$ coronal sections following animal sacrifice. Sections were stained with $1 \%$ TTC solution (Sigma-Aldrich; Merck KGaA) at $37^{\circ} \mathrm{C}$ and incubated in the dark for $30 \mathrm{~min}$. After staining, the sections were fixed with $10 \%$ formalin at $4^{\circ} \mathrm{C}$ for $24 \mathrm{~h}$. Healthy myocardial tissues were stained brick-red and the infarcted myocardial tissues were white. A digital camera was used to capture images. Image J software (version 1.8.0; National Institutes of Health) was used to calculate myocardial infarction size.

Hematoxylin and eosin (HE) staining. Ischemic myocardial tissues from each group were collected and fixed using $4 \%$ paraformaldehyde at $4^{\circ} \mathrm{C}$ for $24 \mathrm{~h}$, embedded in paraffin, and sliced into $4 \mu \mathrm{m}$ sections. Sections were then dewaxed with xylene, washed twice with anhydrous ethanol, dehydrated using an ethanol gradient, stained with hematoxylin for $5 \mathrm{~min}$ and washed with double-distilled water. Subsequently, at room temperature, the sections were differentiated with $0.5 \%$ alcohol hydrochloride for $30 \mathrm{sec}$, stained with eosin for $2 \mathrm{~min}$, washed with xylene for $2 \mathrm{~min}$ and sealed with neutral resin. Finally, the sections were observed under a light microscope.

Cell culture. The rat cardiomyocyte H9C2 and 293T cell lines (American Type Culture Collection) was cultured in Dulbecco's modified Eagle's medium (Gibco; Thermo Fisher Scientific, Inc.) containing 10\% fetal bovine serum (Gibco; Thermo Fisher Scientific, Inc.), $100 \mathrm{mg} / \mathrm{ml}$ penicillin and $100 \mathrm{mg} / \mathrm{ml}$ streptomycin in a humidified incubator at $37^{\circ} \mathrm{C}$ containing $5 \% \mathrm{CO}_{2}$ and $95 \% \mathrm{O}_{2}$. Subsequent experimentation was carried out at $50 \%$ confluency.

Cell Counting Kit-8 (CCK-8). H9C2 cells were collected and treated with various concentrations of propofol $(0,6.25,12.5$, $25,50,100$ and $200 \mu \mathrm{M})$ at $37^{\circ} \mathrm{C}$ for $6 \mathrm{~h}$ to determine propofol cytotoxicity. The cells were seeded into 96-well plates 
$\left(3,000\right.$ cells/well) and cultured at $37^{\circ} \mathrm{C}$. After culture for 0,24 , 48 and $72 \mathrm{~h}$, samples were incubated with $10 \mu \mathrm{l} \mathrm{CCK}-8$ reagent (Beyotime Institute of Biotechnology). Following incubation at $37^{\circ} \mathrm{C}$ for $4 \mathrm{~h}$, the optical density was measured at $450 \mathrm{~nm}$ and the cell proliferation curve was drawn.

Establishment of the HG hypoxia/reperfusion $(H / R)$ model. For hypoxia, $\mathrm{H} 9 \mathrm{C} 2$ cells were incubated in $94 \% \mathrm{~N}_{2}, 5 \% \mathrm{CO}_{2}$ and $1 \% \mathrm{O}_{2}$ at $37^{\circ} \mathrm{C}$ for $12 \mathrm{~h}$, and then reoxygenated for $6 \mathrm{~h}(9,19)$. Cells were treated with $0,6.25,12.5,25,50,100$ and $200 \mu \mathrm{M}$ propofol following $12 \mathrm{~h}$ of hypoxia and then propofol treatment and reoxygenation were simultaneously performed for $6 \mathrm{~h}$ at $37^{\circ} \mathrm{C}$.

Cells were assigned into the following groups: the blank group (H9C2 cells without any treatment), the HG group (cultured in $4.5 \mathrm{~g} / \mathrm{l} \mathrm{HG}$ medium without $\mathrm{H} / \mathrm{R}$ treatment), the $\mathrm{HG}+\mathrm{H} / \mathrm{R}$ group (H/R treatment was performed following $48 \mathrm{~h}$ of $\mathrm{HG}$ culture), the $\mathrm{P} 25$ group (H/R treatment + propofol postconditioning was performed following HG culture), the P25 + mimic negative control (NC) group (mimic $\mathrm{NC}+\mathrm{H} / \mathrm{R}+25 \mu \mathrm{M}$ propofol postconditioning was performed following HG culture), the P25 + miR-200c-3p mimic (miR-200C-3p mimic $+\mathrm{H} / \mathrm{R}+25 \mu \mathrm{M}$ propofol postconditioning was performed following $\mathrm{HG}$ culture), the P25 + small interfering RNA (si)-NC group (si-NC $+\mathrm{H} / \mathrm{R}+25 \mu \mathrm{M}$ propofol postconditioning was performed following $\mathrm{HG}$ culture) and the P25 + si-AdipoR2 group (si-Adipor2 + H/R $+25 \mu \mathrm{M}$ propofol postconditioning was performed following HG culture). miR-200c-3p mimic, mimic NC, si-Adipor2, and si-NC were designed by Shanghai GenePharma Co., Ltd. The 293T cells (American Type Culture Collection) were transfected with mimic NC, miR-200c-3p mimic, si-NC, or si-AdipoR2 (Shanghai Genechem Co., Ltd.; $30 \mathrm{nM}$ miRNA-mimic and 40-100 nM siRNA) using Lipofectamine $2000^{\circledR}$ (Invitrogen; Thermo Fisher Scientific, Inc.). The sequence of the miR-200c-3p mimic was 5'-UAAUACUGC CGGGUAAUGAUG-3' and the sequence of si-AdipoR2 was 5'-GCATCAAGCTGTACATATA-3', the sequence of mimic NC was 5'-AUGUCCGUAAGGAUACUGGUA-3' and the sequence of si-NC was 5'-TCGCACTAATAAGGTACAT-3'. miR-200c-3p mimic, si-AdipoR2 and their controls were transfected into the target cells using the Lipofectamine RNAiMAX Transfection Kit (Invitrogen; Thermo Fisher Scientific, Inc.) at $37^{\circ} \mathrm{C}$ for $48 \mathrm{~h}$ according to the manufacturer's instructions. Prior to transfection, the cells were seeded into 6 -well plates at $1 \times 10^{6}$ cells/well. On the day of transfection, Lipofectamine RNAiMAX transfection reagent and opti-MEM culture medium (Thermo Fisher Scientific, Inc.) were at room temperature for $10 \mathrm{~min}$ and added into the 6-well plates. After $24 \mathrm{~h}$, the transfection efficiency in $\mathrm{H} / \mathrm{R}$ cells was verified by reverse transcription-quantitative PCR (Fig. S1) and other experiments were performed.

Dual-luciferase reporter assay. The binding sites of miR-200c-3p and AdipoR2 were predicted using the TargetScan bioinformatics database v. 7.1 (http://www. targetscan.org/vert71/) (20). Subsequently, the wild-type (Wt) and mutant (Mut) 3'untranslated region of the AdipoR2 and miR-200c-3p binding site sequences were amplified and cloned into the pmirGLO dual-luciferase miRNA target expression vector (Promega Corp.). The Wt plasmid AdipoR2-Wt and the corresponding Mut plasmid AdipoR2-Mut were constructed and co-transfected with mimic NC or miR-200c-3p mimic into $293 \mathrm{~T}$ cells at a density of $2 \times 10^{4}$ using Lipofectamine 2000 reagent according to the manufacturer's protocol. After $48 \mathrm{~h}$ of the transfection at room temperature, the luciferase activity was detected using the Dual-Luciferase Reporter Assay System (Promega Corp.) according to the manufacturer's instructions, with Renilla luciferase used for normalization. The fluorescence intensity was normalized to the mimic NC plasmid group.

ROS detection. The ROS Assay kit (Nanjing Jiancheng Bioengineering Institute) was used for ROS detection according to the manufacturer's protocol. Briefly, myocardial tissue homogenate or cells were collected and suspended in diluted 2,7-dichlorodi-hydrofluorescein diacetate, incubated at $37^{\circ} \mathrm{C}$ for $30 \mathrm{~min}$ and centrifuged at $1,000 \mathrm{x} \mathrm{g}$ at $37^{\circ} \mathrm{C}$ for 5 min to obtain the precipitates. Precipitates were suspended in phosphate buffer saline (PBS) and observed under a confocal microscope (LSM 510 Meta microscope; Zeiss GmbH) using an emission wavelength of $525 \mathrm{~nm}$.

Detection of related biochemical indexes. Following the reperfusion procedure, after euthanasia by intraperitoneal injection of $2 \%$ pentobarbital sodium $(200 \mathrm{mg} / \mathrm{kg}), 1 \mathrm{ml}$ femoral arterial blood was collected and centrifuged at $300 \mathrm{x} \mathrm{g}$ at $4^{\circ} \mathrm{C}$ for $15 \mathrm{~min}$ and the supernatant was extracted. The $\mathrm{H} 9 \mathrm{C} 2$ cell line was also used in this experiment. Creatinine kinase-myocardial band levels (CK-MB) were subsequently detected using the Rat CK-MB Isoenzyme ELISA kit (cat. no. E02C0085; BlueGene Biotech) according to the manufacturer's protocol. Lactate dehydrogenase (LHD) levels were determined using a LDH Assay Kit (cat. no. A020-2-2; Nanjing Jiancheng Bioengineering Institute) according to the manufacturer's instructions. Similarly, SOD levels were quantified using a Total SOD Assay kit (water-soluble tetrazolium-8 method; cat. no. S0101S; Beyotime Institute of Biotechnology), and MDA levels were detected using a Lipid Peroxidation MDA Assay kit (cat. no. S0131S; Beyotime Institute of Biotechnology) according to the manufacturer's protocol. Bax and B cell lymphoma-2 (BCL-2) levels were detected using a Rat Bax ELISA kit (cat. no. E-EL-R0098; Elabscience Biotechnology, Inc.) or a Rat Bcl-2 ELISA kit (E-EL-R0096; Elabscience Biotechnology, Inc.) according to the manufacturer's protocol. Absorbance values were measured at the corresponding wavelengths.

TdT-mediated dUTP Nick-End Labeling (TUNEL) assay. Myocardial tissues were fixed with $4 \%$ paraformaldehyde at room temperature for $24 \mathrm{~h}$. Cardiomyocyte apoptosis was detected using the One-Step TUNEL Cell Apoptosis Detection kit (cat. no. C1088; Beyotime Institute of Biotechnology) on $4 \mu \mathrm{m}$-thick cardiac sections or $\mathrm{H} 9 \mathrm{C} 2$ cells. Briefly, the treated sections or cells were incubated with $50 \mu 1$ TUNEL reagent at $37^{\circ} \mathrm{C}$ for $1 \mathrm{~h}$, rinsed three times with PBS and counterstained with 4',6-diamidino-2-phenylindole (DAPI; $1 \mathrm{mg} / \mathrm{ml}$ ) at room temperature in the dark for $5 \mathrm{~min}$. The cells $\left(1 \times 10^{6}\right)$ were collected and sealed with anti-fluorescence quenching sealing solution as mounting medium (Beyotime Institute 
of Biotechnology). Following another three PBS washes, the sections or cells were observed under a fluorescence microscope (Leica Microsystems $\mathrm{GmbH}$ ). The percentage of apoptotic cells was calculated using five randomly selected fields. The cell apoptotic rate=the number of apoptotic cells (green)/total cells (blue) x100.

Reverse transcription-quantitative PCR (RT-qPCR). Total RNA was extracted from myocardial tissues or H9C2 cells using TRIzol ${ }^{\circledR}$ reagent (Invitrogen; Thermo Fisher Scientific, Inc.) according to the manufacturer's instructions. Total RNA was reverse transcribed using the Taqman Micro-RNA Reverse Transcription Reagents kit (Thermo Fisher Scientific, Inc.) according to the manufacturer's instructions. qPCR was performed using the SYBR Green Real-Time PCR Master mix (Thermo Fisher Scientific, Inc.) according to the manufacturer's protocol. The following thermocycling conditions were used for qPCR: initial denaturation at $95^{\circ} \mathrm{C}$ for $5 \mathrm{~min}$; 40 cycles of denaturation at $95^{\circ} \mathrm{C}$ for $15 \mathrm{sec}$, annealing at $58^{\circ} \mathrm{C}$ for $35 \mathrm{sec}$, elongation at $72^{\circ} \mathrm{C}$ for $30 \mathrm{sec}$; and final extension at $72^{\circ} \mathrm{C}$ for $10 \mathrm{~min}$. Expression levels were quantified using the $2^{-\Delta \Delta \mathrm{Cq}}$ method (21) and normalized to the internal reference genes, GAPDH and U6. Primer sequences are shown in Table I.

Western blotting. Total protein was extracted from myocardial tissues or H9C2 cells using radio-immunoprecipitation assay (RIPA) buffer (Beyotime Institute of Biotechnology). Total protein was quantified using a Bicinchoninic Acid (BCA) Protein Concentration Determination kit (Beyotime Institute of Biotechnology). Subsequently, proteins $(20 \mu \mathrm{g})$ were bathed in boiling water for $5 \mathrm{~min}$. Proteins were separated by SDS-PAGE on a $15 \%$ gel following denaturation. Proteins were transferred onto polyvinylidene fluoride membranes using the wet-transfer method, blocked with 5\% skimmed milk-TBS-Tween 20 (TBST; containing $0.05 \%$ Tween-20) at room temperature for $60 \mathrm{~min}$. The samples were developed using ECL working fluid (MilliporeSigma). Membranes were then washed using TBST and incubated with the primary antibodies phosphorylated-STAT3 (88 kDa; 1:20,000; cat. no. ab76315; Abcam), STAT3 (88 kDa; 1:1,000; cat. no. ab68153; Abcam) and GAPDH (36 kDa; 1:1,000; cat. no. ab8245; Abcam) overnight at $4^{\circ} \mathrm{C}$. Membranes were washed with TBST. Following the primary incubation, membranes were incubated with the secondary goat anti-rabbit $\operatorname{IgG}$ (heavy chain and light chain; HRP; 1:1,000; cat. no. ab205718; Abcam) at $37^{\circ} \mathrm{C}$ for $2 \mathrm{~h}$. A chemiluminescence method was used for visualization and Image J software (version 1.52a; National Institutes of Health) was used for gray value analysis. GAPDH was used as the loading control.

Statistical analysis. SPSS 21.0 statistical software (IBM Corp.) and GraphPad Prism 8.0.1 (GraphPad Software, Inc.) were used for data analysis. Cell experiments were repeated three times. The Shapiro-Wilk test demonstrated that all data were normal distributed. All data are expressed as the mean \pm standard deviation. Unpaired student's t-test was used to analyze comparisons between two groups, whereas one-way analysis of variance was used to analyze comparisons between more than two groups followed by Tukey's post-hoc test. $\mathrm{P}<0.05$ was considered to indicated a statistically significant difference.
Table I. Sequences of primers used for reverse transcription-quantitative PCR.

\begin{tabular}{ll}
\hline Gene & \multicolumn{1}{c}{ Sequence $\left(5^{\prime} \rightarrow 3^{\prime}\right)$} \\
\hline miR-200c-3p & F: TAATACTGCCGGGTAAT \\
R: TGTCGTGGAGTCGGC \\
FdipoR2 & F: ATGAACGAGCCAACCGAACA \\
& F: TCAGAAGAGCAATACCAGAG \\
U6 & R: AAATATGGAACGCTTCACGA \\
GAPDH & F: ATGGTGAAGGTCGGTGTG \\
& R: TCACCCCATTTGATGTTA \\
\hline
\end{tabular}

miR, microRNA; AdipoR2, adiponectin receptor 2; F, forward; $R$, reverse.

\section{Results}

Propofol postconditioning has a protective effect on diabetic $M I / R I$. To verify the effect of propofol postconditioning on diabetic MI/RI, diabetic MI/RI rat models were established and the infarct size was detected using TTC staining. There was no infarct in the myocardium in the DS group. The results demonstrated that the infarct size of the DI group was significantly increased compared with the DP group (Fig. 1A; $\mathrm{P}<0.01)$. The results of $\mathrm{HE}$ staining demonstrated that in the DS group, rat cardiac structure and cell morphology were normal, the myocardial cells were organized, the capsule was intact and no inflammatory cell or red blood cell infiltration was observed in the stroma. However, in the DI group there was clear myocardial infarction, disordered arrangement of myocardial cells, widened myocardial fiber gap and inflammatory cell infiltration in the stroma. All these conditions were markedly improved in the DP group (Fig. 1B). Furthermore, it has been reported that propofol can relieve I/R injury by reducing oxidative stress and suppressing apoptosis (10). Therefore, oxidative stress indexes and apoptosis in the various groups were detected. In the DI group, ROS, CK-MB, MDA and LDH levels were significantly upregulated, whereas SOD levels were significantly reduced, all compared with the DP group (Fig. 1C-G; all $\mathrm{P}<0.01$ ). Moreover, the results of TUNEL staining demonstrated that compared with the DI group, the apoptosis of myocardial tissue was significantly downregulated in the DP group (Fig. $1 \mathrm{H} ; \mathrm{P}<0.01$ ). Bax and Bcl-2 levels in serum were also detected using ELISA. Compared with the DI group, Bax was significantly downregulated and Bcl-2 was significantly upregulated in the DP group (Fig. 1I; $\mathrm{P}<0.01)$. Therefore, these findings suggested that propofol postconditioning may inhibit oxidative stress and apoptosis, thus improving MI/RI.

Propofol postconditioning has a protective effect on $\mathrm{H} 9 \mathrm{C} 2$ cells induced by $H G$ and $H / R$. The effect of propofol postconditioning using in vitro models was also investigated. The CCK-8 assay was performed to detect the effect of propofol on $\mathrm{H} 9 \mathrm{C} 2$ cells. The results demonstrated that $\mathrm{H} 9 \mathrm{C} 2$ cells treated with $25 \mu \mathrm{M}$ propofol had a significantly higher cell viability 
A
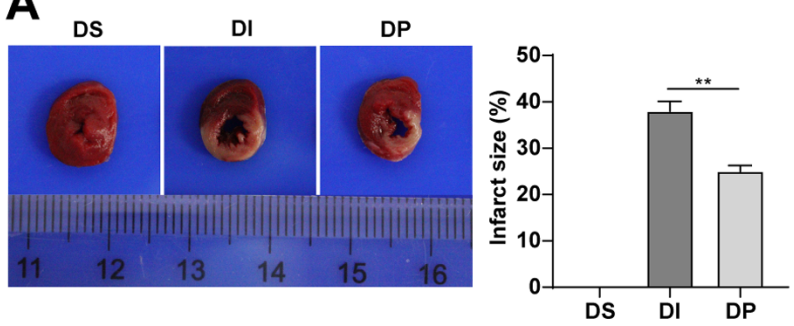

C

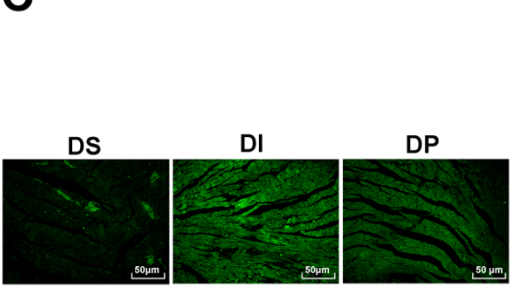

H

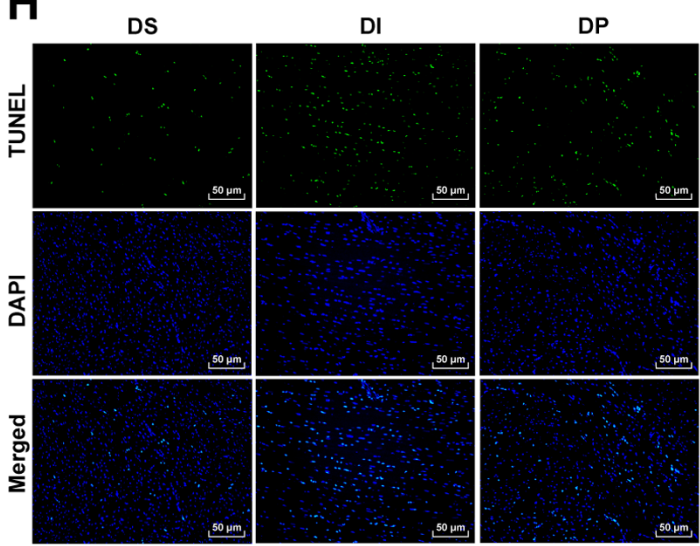

G

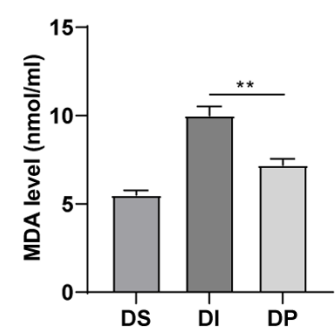

B

DS

DI

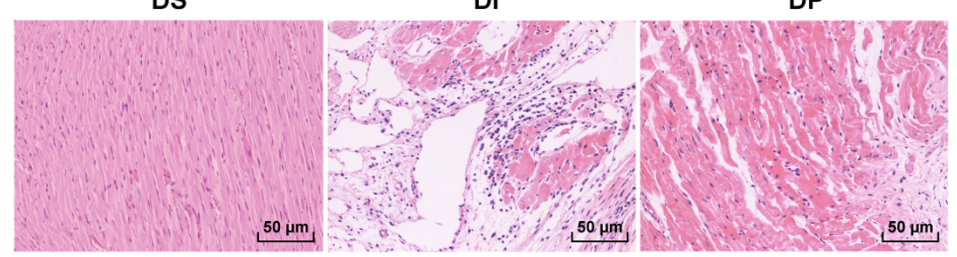

F

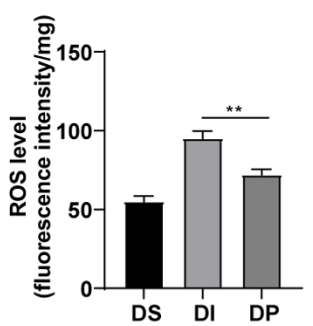

E

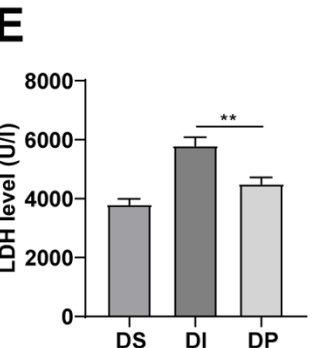

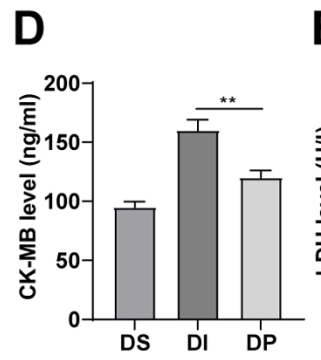

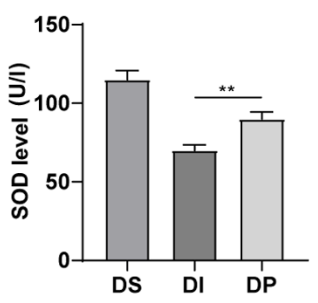

I

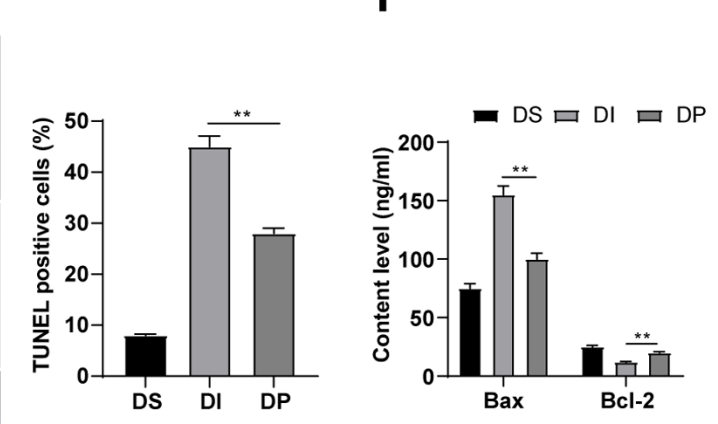

\section{(}

Figure 1. Propofol postconditioning has protective effect on diabetic MI/RI. (A) Area of myocardial infarction was detected using 2,3,5-triphenyl-2H-tetrazolium chloride staining. (B) Pathological changes of MI/RI tissue were detected using HE staining. Oxidative stress index levels were detected using ELISA: (C) ROS, (D) CK-MB, (E) LDH, (F) SOD and (G) MDA levels. (H) Apoptosis was detected using TUNEL staining. (I) Bax and Bcl-2 serum levels were detected using ELISA. $\mathrm{n}=6$. Three independent cell tests were performed. Data are presented as the mean \pm standard deviation. One-way ANOVA was used for comparisons between groups followed by Tukey's post hoc test. ${ }^{* *} \mathrm{P}<0.01$. MI/RI, myocardial ischemia reperfusion injury; ROS, reactive oxygen species; CK-MB, creatinine kinase-myocardial band; LDH, lactate dehydrogenase; SOD, superoxide dismutase; MDA, malondialdehyde; DS, sham group; DI, MI/RI group; DP, MI/RI + propofol group.

compared with $0 \mu \mathrm{M}$ concentrations (Fig. $2 \mathrm{~A} ; \mathrm{P}<0.05$ ). Therefore, a propofol concentration of $25 \mu \mathrm{M}$ was used to perform postconditioning on $\mathrm{H} 9 \mathrm{C} 2$ cells induced by $\mathrm{HG}$ and $\mathrm{H} / \mathrm{R}$. Following propofol postconditioning, ROS, CK-MB, MDA and LDH levels were significantly reduced and SOD levels were significantly increased compared with the $\mathrm{HG}+$ H/R group (Fig. 2B-F; P<0.01). Furthermore, the results of the TUNEL assay demonstrated that propofol postconditioning significantly suppressed apoptosis compared with the HG + H/R group (Fig. 2G; $\mathrm{P}<0.01$ ). Compared with the $\mathrm{HG}+\mathrm{H} / \mathrm{R}$ group, it was also observed that Bax levels were significantly reduced and $\mathrm{Bcl}-2$ levels were significantly upregulated as a result of propofol postconditioning (Fig. $2 \mathrm{H} ; \mathrm{P}<0.05$ ). These findings suggested that propofol postconditioning may protect $\mathrm{H} 9 \mathrm{C} 2$ cells from $\mathrm{HG}$ and $\mathrm{H} / \mathrm{R}$.

Propofol postconditioning demonstrates antioxidant and antiapoptotic effects on H9C2 cells by inhibiting $m i R-200 c-3 p$. Previous studies indicate that propofol exerts its protective effects on $\mathrm{I} / \mathrm{R}$ injury via miRNAs $(11,13)$. Among them, miR-200c is known to promote an oxidative reaction and is associated with diabetic MI/RI (14). Subsequently, the expression patterns of miR-200c-3p were detected using RT-qPCR, which demonstrated that miR-200c-3p expression levels were significantly upregulated in in vivo and in vitro models, compared with the propofol postconditioning groups which exhibited significantly downregulated miR-200c-3p expression levels (Fig. 3A-B; all $\mathrm{P}<0.01$ ). Moreover, miR-200c-3p expression levels were significantly increased using miR-200c-3p mimic to further investigate the effect of propofol on miR-200c-3p compared with the P25 + mimics NC group $(\mathrm{P}<0.01)$. ROS, CK-MB, MDA and LDH levels were significantly upregulated and SOD levels were significantly downregulated as a result of miR-200c-3p overexpression, compared with P25 + mimics NC (Fig. 3C-G; all P<0.01). Furthermore, TUNEL assay 
A

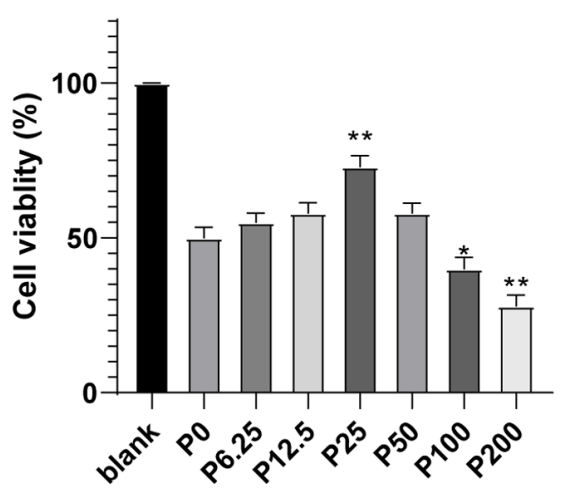

B

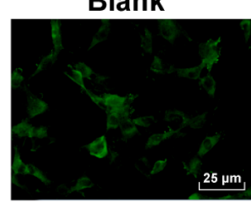

$H G+H / R$

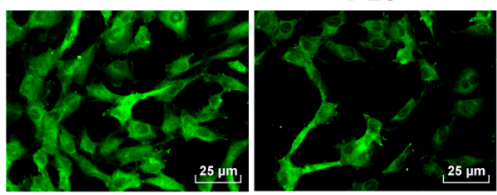

E

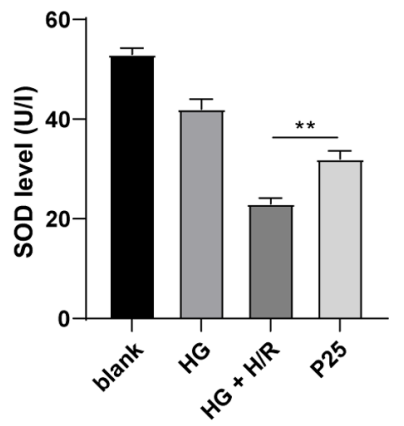

HG

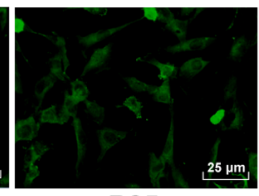

P25
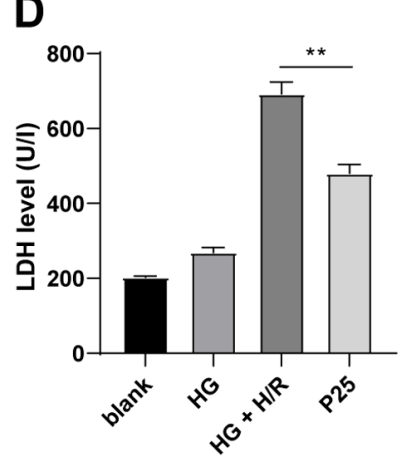

$\mathbf{P} 25$

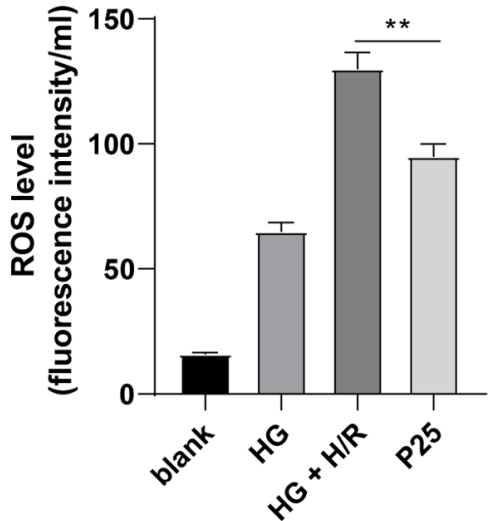

$\mathbf{F}$

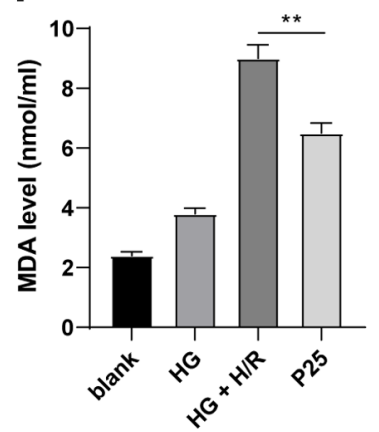

H
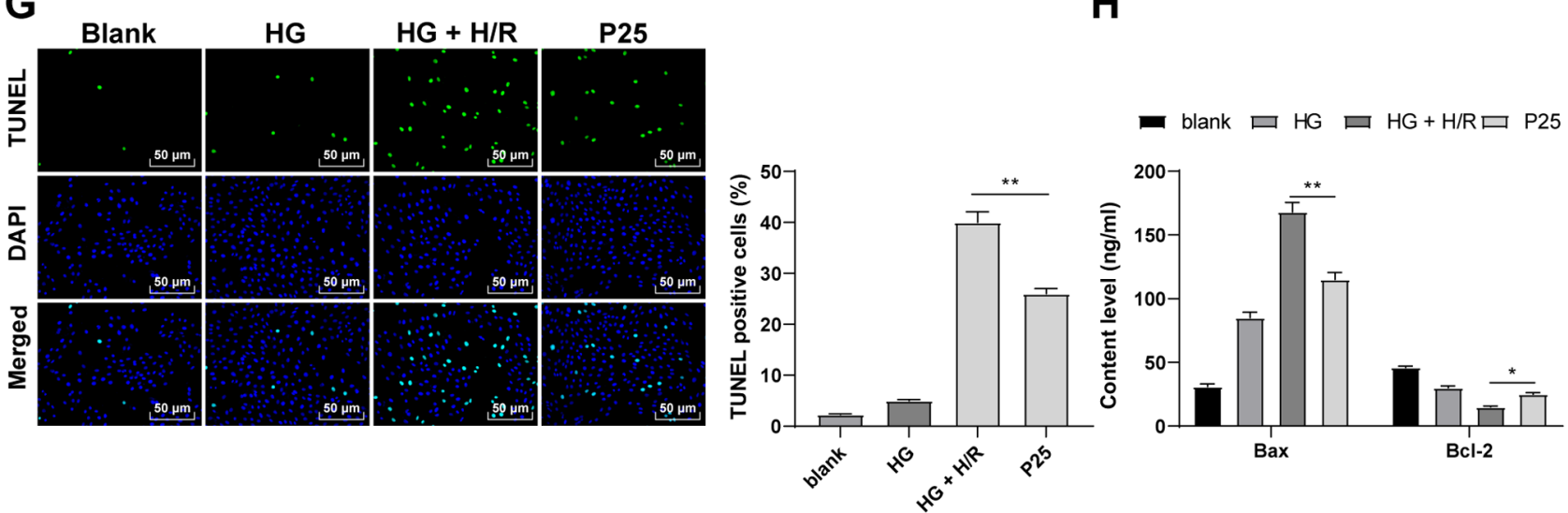

Figure 2. Propofol postconditioning has a protective effect on HG and H/R-induced H9C2 cells. (A) Cell Counting Kit-8 assay was used to detect the toxicity of $\mathrm{H} 9 \mathrm{C} 2$ cells. The results determined that cell activity was significantly higher at a propofol concentration of $25 \mu \mathrm{M}$ compared with the P0 group. Therefore, $25 \mu \mathrm{M}$ propofol was used in subsequent experiments. Oxidative stress index levels were detected using ELISA: (B) ROS, (C) CK-MB, (D) LDH, (E) SOD and (F) MDA. (G) H9C2 cell apoptosis was detected using TUNEL staining. (H) Bax and Bcl-2 levels in H9C2 cells were detected using ELISA. Three independent cell tests were performed. Data are presented as the mean \pm standard deviation. One-way ANOVA was used for comparisons between groups followed by Tukey's post hoc test. " $\mathrm{P}<0.05$ and ${ }^{* *} \mathrm{P}<0.01$. HG, high glucose; $\mathrm{H} / \mathrm{R}$, hypoxia/reperfusion; $\mathrm{P}, \mu \mathrm{M}$ propofol; ROS, reactive oxygen species; CK-MB, creatinine kinase-myocardial band; LDH, lactate dehydrogenase; SOD, superoxide dismutase; MDA, malondialdehyde.

results demonstrated that overexpression of miR-200c-3p significantly increased cell apoptosis compared with P25 + mimics NC (Fig. 3H and I; all $\mathrm{P}<0.05$ ). Overall, these findings indicated that overexpression of miR-200c-3p may partially reverse the antioxidant and antiapoptotic effects of propofol postconditioning.

miR-200c-3p targets AdipoR2. The targeted mRNA of miR-200c-3p was predicted and analyzed using the TargetScan database (20) to explore the downstream molecular mechanism of miR-200c-3p. Among the predicted targets, AdipoR2 was previously associated with the recovery of diabetic MI/RI (22). Therefore, the binding sites between AdipoR2 and miR-200c-3p were predicted using the TargetScan database (Fig. 4A). Subsequently, the binding relationship was verified using the dual-luciferase reporter assay. Compared with the control group, following transfection with miR-200c-3p mimic in the AdipoR2-WT group, the relative luciferase activity was significantly decreased, while that of the AdipoR2-MUT group did not change (Fig. 4B; $\mathrm{P}<0.01$ ). AdipoR 2 mRNA expression levels were detected by RT-qPCR. The results demonstrated that propofol postconditioning significantly upregulated the 

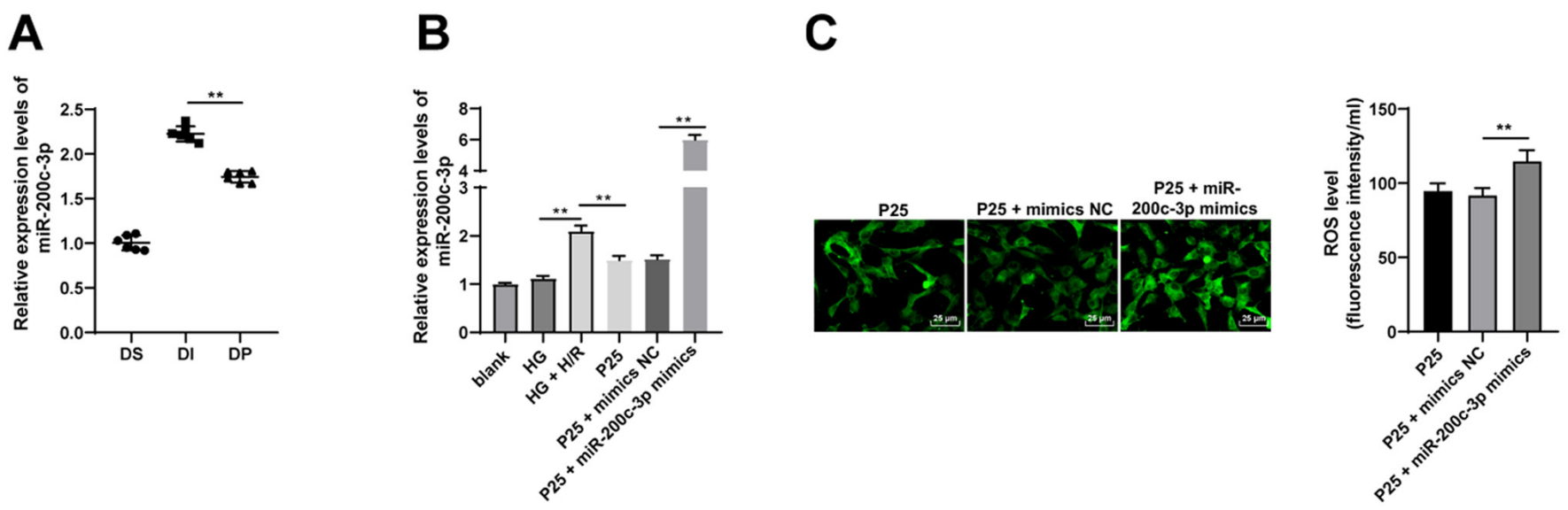

D

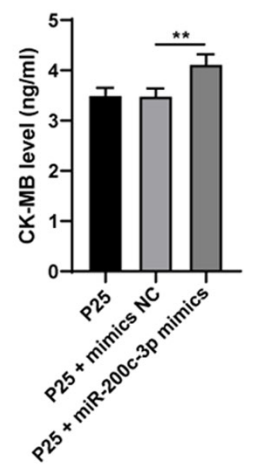

E

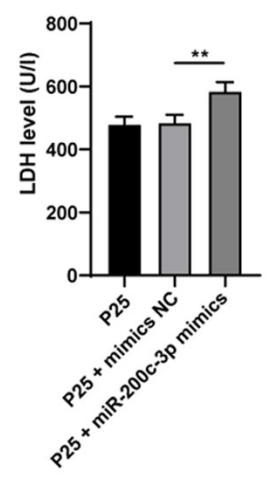

$\mathbf{F}$

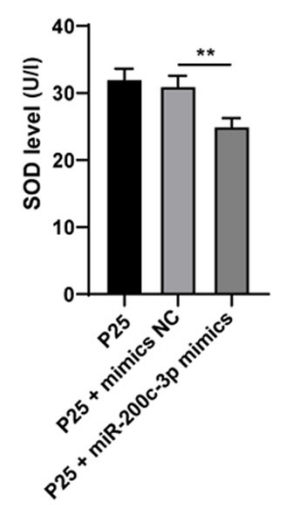

G

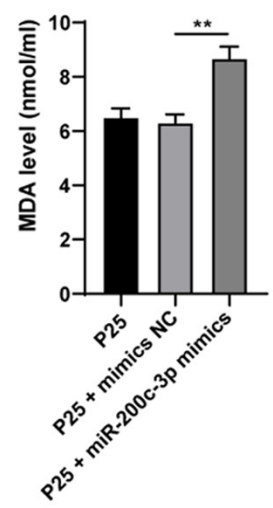

H

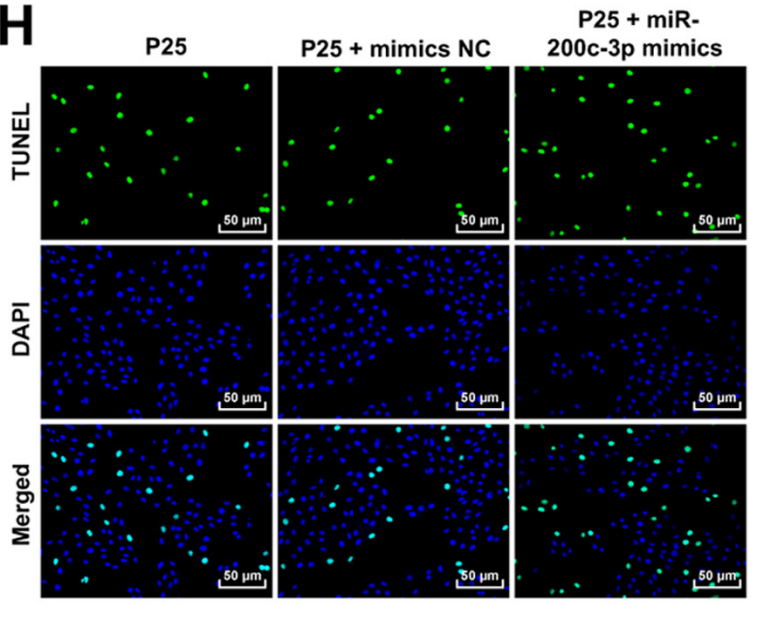

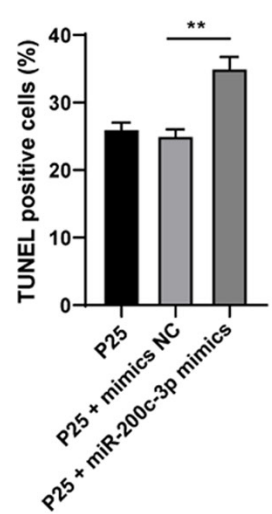

- P25 口 P25+mimics NC

D P25+ miR-200c-3p mimics

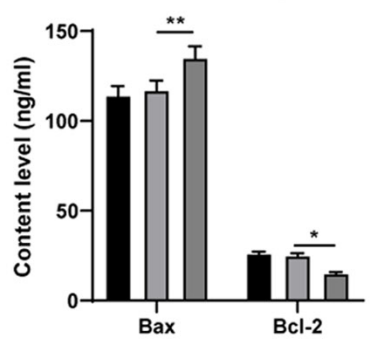

Figure 3. Propofol postconditioning has antioxidant and antiapoptotic effects on H9C2 cells via miR-200c-3p inhibition. miR-200c-3p mimic was transfected into P25-treated cells and mimics NC was used as control. (A) miR-200c-3p expression levels in the diabetic MI/RI rat model were detected by RT-qPCR, $\mathrm{n}=6$. (B) miR-200c-3p expression levels in H9C2 cells induced by HG and H/R were detected using RT-qPCR. Oxidative stress index levels were detected using ELISA: (C) ROS, (D) CK-MB, (E) LDH, (F) SOD and (G) MDA. (H) H9C2 cell apoptosis was detected using TUNEL staining. (I) Bax and Bcl-2 levels in H9C2 cells were detected using ELISA. Three independent cell tests were performed. Data are presented as the mean \pm standard deviation. One-way ANOVA was used for comparisons between groups followed by Tukey's post hoc test. ${ }^{*} \mathrm{P}<0.05$ and ${ }^{* *} \mathrm{P}<0.01$. miR, microRNA; 25 , $25 \mu \mathrm{M}$ propofol; NC, negative control; MI/RI, myocardial ischemic reperfusion; RT-qPCR, reverse transcription-quantitative PCR; ROS, reactive oxygen species; CK-MB, creatinine kinase-myocardial band; LDH, lactate dehydrogenase; SOD, superoxide dismutase; MDA, malondialdehyde; DS, sham group; DI, MI/RI group; DP, MI/RI + propofol group; HG, high glucose; H/R, hypoxia/reperfusion. 
A

\begin{tabular}{|c|c|c|}
\hline $\begin{array}{l}\text { Position } 2535-2541 \text { of ADIPOR2 3' UTR } \\
\text { rno-miR-200c-3p }\end{array}$ & 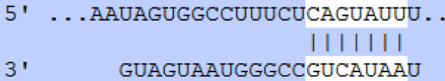 & $\begin{array}{c}7 \mathrm{mer}- \\
\mathrm{m} 8\end{array}$ \\
\hline
\end{tabular}
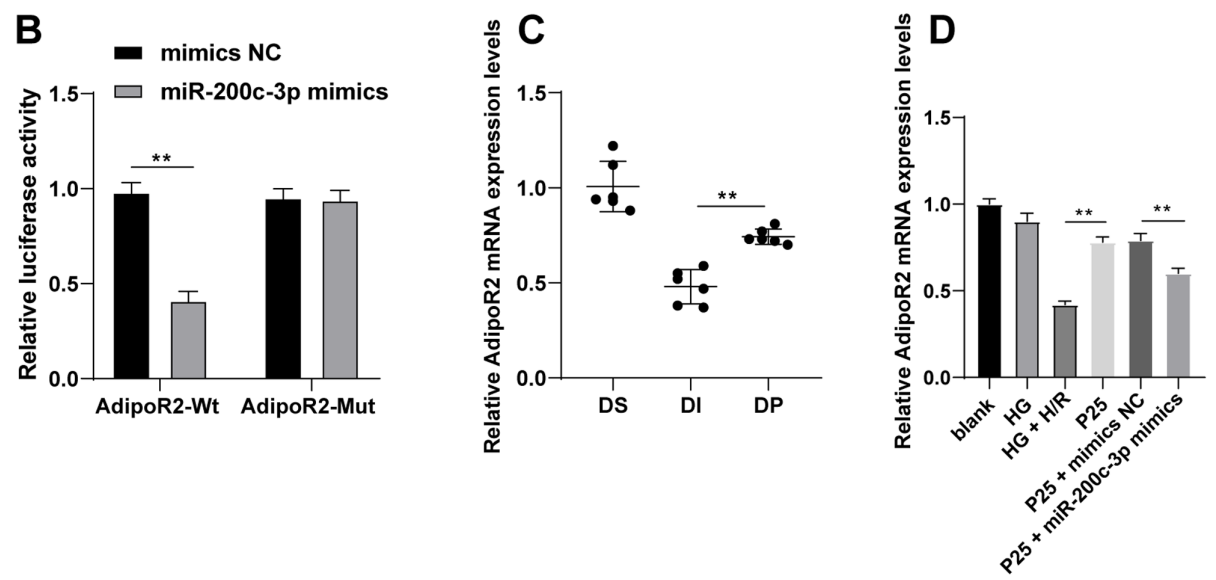

Figure 4. Propofol postconditioning upregulates AdipoR2 and downregulates miR-200c-3p. (A) Binding sites of miR-200c-3p and AdipoR2 were predicted using TargetScan. (B) Dual-luciferase reporter assay was used to detect the targeting relationship between miR-200c-3p and AdipoR2. (C) AdipoR2 mRNA expression levels in diabetic myocardial ischemic reperfusion rat model following propofol postconditioning were detected by RT-qPCR, $\mathrm{n}=6$. (D) AdipoR2 mRNA expression levels in $\mathrm{H} 9 \mathrm{C} 2$ cells following propofol postconditioning were detected by RT-qPCR. Three independent cell tests were performed. Data are presented as the mean \pm standard deviation. Unpaired Student's t-test was used for comparisons between two groups in panel B, and one-way ANOVA followed by Tukey's post hoc test was used for comparisons between multiple groups in panels $\mathrm{C}$ and $\mathrm{D}$. ${ }^{* *} \mathrm{P}<0.01$. AdipoR2, adiponectin receptor 2; miR, microRNA; MI/RI; RT-qPCR, reverse transcription-quantitative PCR; UTR, untranslated region; NC, negative control; P25, $25 \mu \mathrm{M}$ propofol; Wt, wild-type; Mut, mutant; DS, sham group; DI, MI/RI group; DP, MI/RI + propofol group; HG, high glucose; H/R, hypoxia/reperfusion; rno, Rattus norvegicus.

A

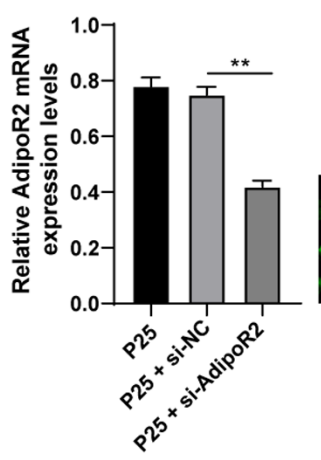

B

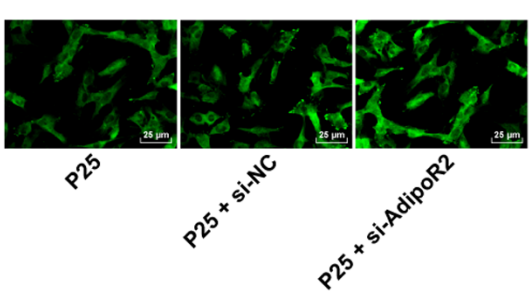

C

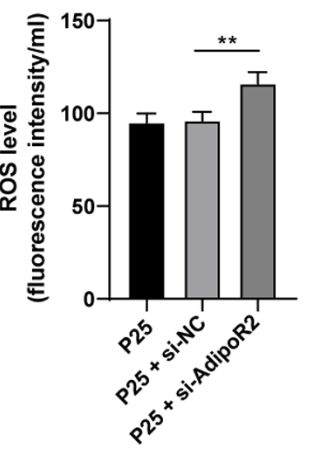

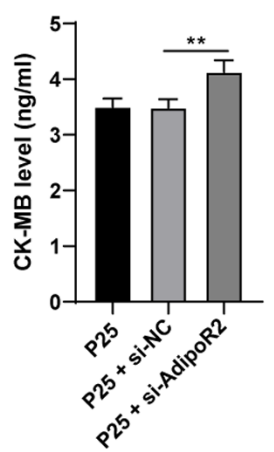

D

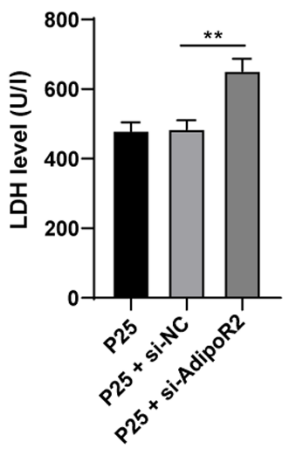

E

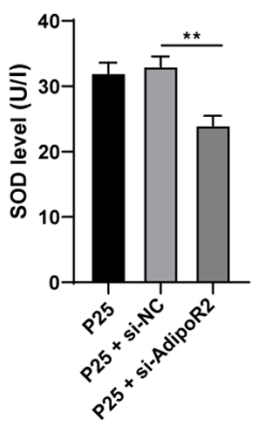

F

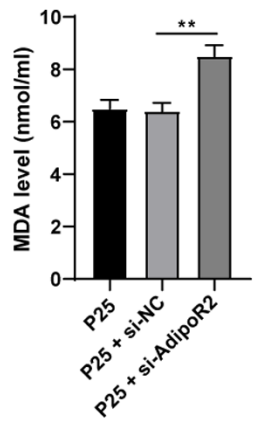

G

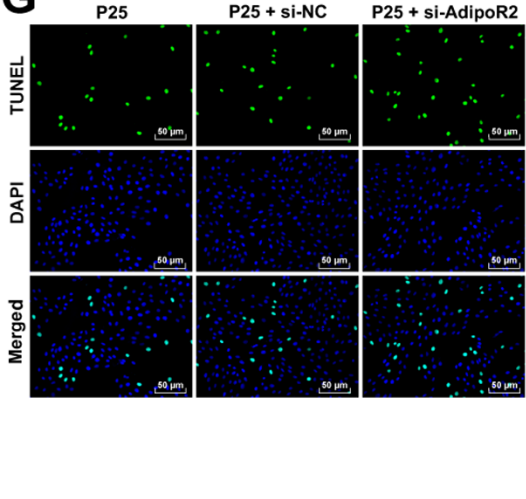

H

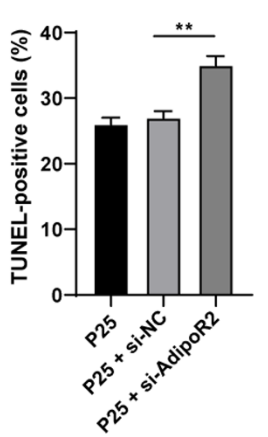

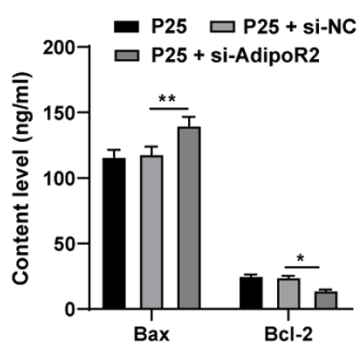

Figure 5. Silencing AdipoR2 partially reverses the antioxidant and antiapoptotic effects of propofol postconditioning on myocardial ischemic reperfusion injury. si-AdipoR2 was transfected into P25-treated cells and si-NC was used as control. (A) AdipoR2 mRNA expression levels in H9C2 cells were detected using reverse transcription-quantitative PCR. Oxidative stress index levels were detected using ELISA: (B) ROS, (C) CK-MB, (D) LDH, (E) SOD and (F) MDA. (G) $\mathrm{H} 9 \mathrm{C} 2$ cell apoptosis was detected using TUNEL staining. (H) Bax and Bcl-2 levels in $\mathrm{H} 9 \mathrm{C} 2$ cells were detected using ELISA. Three independent cell tests were performed. Data are presented as the mean \pm standard deviation. One-way ANOVA was used for comparisons between groups followed by Tukey's post hoc test. " $\mathrm{P}<0.05$ and ${ }^{* *} \mathrm{P}<0.01$. AdipoR2, adiponectin receptor 2; si, small interfering RNA; P25, $25 \mu \mathrm{M}$ propofol; NC, negative control; ROS, reactive oxygen species; CK-MB, creatinine kinase-myocardial band; LDH, lactate dehydrogenase; SOD, superoxide dismutase; MDA, malondialdehyde. 
A

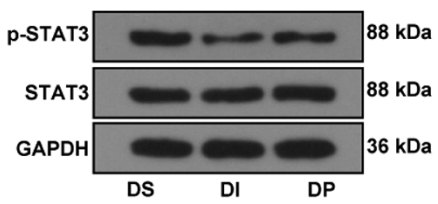

B

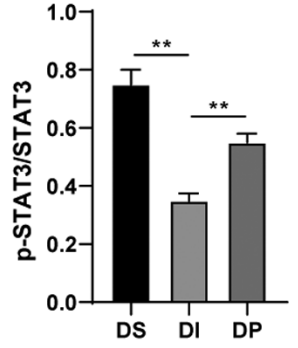

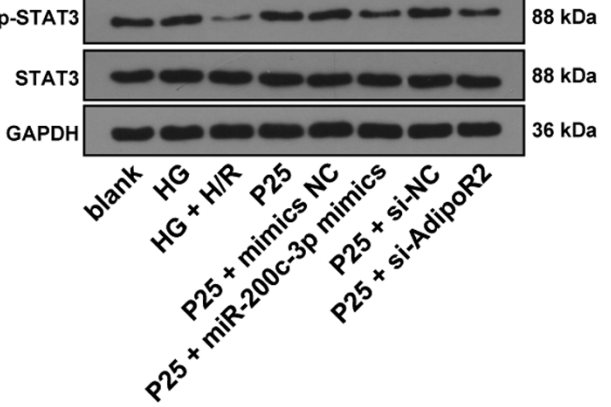

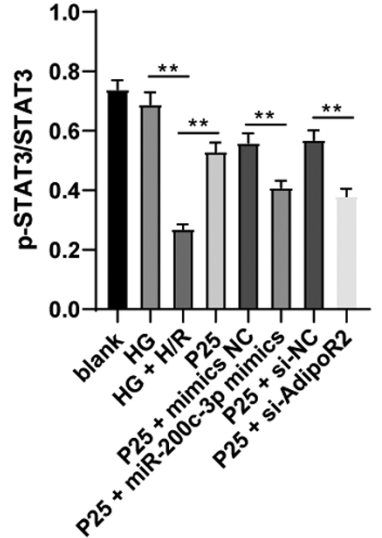

Figure 6. Propofol postconditioning activates the STAT3 pathway via the miR-200c-3p/AdipoR2 axis. (A) STAT3 protein expression levels were detected via western blotting, $n=6$. (B) STAT3 protein expression levels in the H9C2 cell high sugar H/R model were detected via western blotting. Three independent cell tests were performed. Data are presented as the mean \pm standard deviation. One-way ANOVA was used for comparisons between groups followed by Tukey's post hoc test. ${ }^{* *} \mathrm{P}<0.01$. miR, microRNA; AdipoR2, adiponectin receptor 2; H/R, hypoxia/reperfusion; DS, sham group; DI, MI/RI group; DP, MI/RI + propofol group; HG, high glucose; p, phosphorylated; P25, $25 \mu \mathrm{M}$ propofol; NC, negative control; si, small interfering RNA.

expression of AdipoR 2 in vivo and in vitro compared with the $\mathrm{DI}$ or $\mathrm{HG}+\mathrm{H} / \mathrm{R}$ group, respectively (Fig. $4 \mathrm{C}$ and $\mathrm{D}$; all $\mathrm{P}<0.01$ ). Furthermore, miR-200c-3p overexpression significantly decreased AdipoR2 mRNA expression levels compared with the P25 + mimics NC group. Overall, these results suggested that propofol postconditioning might manipulate MI/RI by downregulating miR-200c-3p expression and upregulating AdipoR2 expression.

AdipoR2 downregulation reverses the antioxidative and antiapoptotic effects of propofol on $\mathrm{H} 9 \mathrm{C} 2$ cells. To further investigate the function of AdipoR2, AdipoR2 expression levels were silenced in P25-treated cells using si-AdipoR2. Compared with the si-NC group, the mRNA expression levels of AdipoR2 were significantly reduced in the P25+ si-AdipoR2 group (Fig. 5A; $\mathrm{P}<0.01$ ). The results demonstrated that ROS, CK-MB, MDA and LDH levels were significantly enhanced and SOD levels were significantly downregulated as a result of AdipoR2 knockdown, compared with P25 + si-NC (Fig. 5B-F; all $\mathrm{P}<0.01$ ). Furthermore, the results of the TUNEL assay and Bax and Bcl-2 ELISAs demonstrated that AdipoR2 silencing significantly increased apoptosis in $\mathrm{H} 9 \mathrm{C} 2$ cells compared with $\mathrm{P} 25+$ si-NC (Fig. 5G and $\mathrm{H}$; all $\mathrm{P}<0.05$ ). Overall, these results indicated that silencing AdipoR2 may partially reverse the antioxidant and antiapoptotic effects of propofol postconditioning on MI/RI.

Propofol postconditioning activated the STAT3 pathway via the miR-200c-3p/AdipoR2 axis. Previous studies have reported that adiponectin (APN) can restore STAT3 activity and suppress apoptosis through AdipoR2 following anoxia of cardiomyocytes $(22,23)$. Therefore, in order to validate whether the STAT3 pathway was involved in the protective effect of propofol postconditioning on diabetic MI/RI, STAT3 protein expression levels were detected via western blotting. The results demonstrated that propofol postconditioning significantly upregulated the phosphorylation levels of STAT3 compared with the DI group. Furthermore, miR-200c-3p overexpression or AdipoR2 knockdown of AdiopR2 significantly reduced STAT3 phosphorylation levels compared with P25 + mimics NC and P25 + si-NC, respectively (Fig. 6A and B; all $\mathrm{P}<0.01)$. These results demonstrated that propofol postconditioning may activate the STAT3 pathway and alleviate MI/RI via the miR-200c-3p/AdipoR 2 axis.

\section{Discussion}

Patients with diabetes are predisposed to MI/RI, which is one of the leading causes of mortality and disability (24). Preconditioning with propofol during the process of reperfusion is known to confer protection against MI/RI (25). Therefore, the present study aimed to elucidate the mechanism of propofol postconditioning on diabetic MI/RI and demonstrated that propofol postconditioning attenuated diabetic MI/RI via the miR-200c-3p/AdipoR2/STAT3 pathway (Fig. S2).

Oxidative stress and apoptosis serve a vital role in the pathogenesis of I/R injury (26). ROS, CK-MB, LDH and SOD are regarded as important indexes of oxidative stress $(27,28)$. Notably, propofol pretreatment was previously indicated to inhibit oxidative stress and further improve nerve function in rats with I/R injury (29). Preconditioning occurs before ischemia. Although propofol preconditioning has been reported to have a protective effect on ischemia-reperfusion in the myocardium and brain, it is often unable to predict the occurrence of ischemia clinically, which limits the effect of preconditioning (30). Postconditioning is applied for the ischemic tissues. It is to establish a more effective reperfusion method to treat and save the ischemic tissues and to minimize reperfusion injury (30). Therefore, postconditioning of propofol may have a greater application as a treatment. Shedding a new light on the effect of propofol postconditioning on oxidative stress in diabetic MI/RI, the present study demonstrated that infarct size was significantly reduced and adverse changes in myocardial cells were markedly reduced following I/R treatment, as a result of propofol postconditioning. Moreover, significantly decreased levels of ROS, CK-MB, MDA and LDH and a significant increase 
in SOD levels were documented upon propofol postconditioning, which suggested reduced oxidative stress. Moreover, Bax and Bcl-2 are widely-used indicators of apoptosis (28). Bax levels were significantly downregulated, whereas Bcl-2 levels were significantly upregulated as a result of propofol postconditioning. Furthermore, propofol has been previously indicated to exert an inhibitory effect on $\mathrm{I} / \mathrm{R}$ injury in a variety of experimental models by suppressing apoptosis and reducing oxidative stress (10). These results demonstrated that propofol postconditioning may protect against diabetic MI/RI by attenuating oxidative stress and apoptosis. The present study also revealed that propofol postconditioning conferred protective effects on $\mathrm{H} 9 \mathrm{C} 2$ cardiomyocytes induced by $\mathrm{HG}$ and $\mathrm{H} / \mathrm{R}$ injury in vitro. A recent study also reported that the cardioprotective effects of propofol against MI/RI are dependent on miRNAs (25). miR-200c was previously shown to exhibit proapoptotic cytotoxicity in tumors and endothelial cells (31). Overexpression of miR-200c is also known to inhibit the protective effect of sevoflurane preconditioning against I/R injury (32). miR-200c is further associated with diabetic MI/RI, possibly through the upsurge of ROS (14). The present study reported that propofol postconditioning downregulated miR-200c-3p expression levels. The present study also demonstrated that in addition to significantly increased cell apoptosis, ROS, CK-MB, MDA and LDH levels were all significantly enhanced, and SOD levels was significantly reduced as a result of miR-200c-3p overexpression in P25-treated H9C2 cells. A previous study demonstrated the rapid upregulation of $\mathrm{miR}-200 \mathrm{c}$ following transient ischemia, leading to transient ischemic injury (31). Upregulation of miR-200c has also been previously associated with oxidative stress $(33,34)$. In view of these findings, it would be plausible to suggest that overexpression of $\mathrm{miR}-200 \mathrm{c}-3 \mathrm{p}$ partially reversed the antioxidant and antiapoptotic effects of propofol postconditioning. Therefore, propofol postconditioning may serve an antioxidant and antiapoptotic role in $\mathrm{H} 9 \mathrm{C} 2$ cells by inhibiting miR-200c-3p.

Prediction analyses performed using the TargetScan database indicated AdipoR2 as a target gene of miR-200c. AdipoR2 is a type of APN, a cardioprotective molecule derived from adipocytes, and its reduction is known to exacerbate diabetic MI/RI (35). The dual-luciferase reporter assay verified the existence of a targeted binding relationship between miR-200c-3p and AdipoR2. Furthermore, this indicated that propofol postconditioning regulated MI/RI by significantly downregulating miR-200c-3p expression levels and significantly upregulating AdipoR2 mRNA expression levels. Moreover, AdipoR2 silencing in P25-treated cells via si-AdipoR2, resulted in ROS, CK-MB, MDA and LDH levels all being significantly elevated and SOD levels being significantly downregulated. Cardiomyocyte apoptosis was also significantly promoted as a result of AdipoR2 silencing. APN is further known to exert protective effects through antiapoptotic activities and antioxidative stress and has been previously indicated as a potential therapy for HI/RI (36). Furthermore, another study illustrated that AdipoR knockdown contributes to aggravating diabetic MI/RI, wherein AdipoR activation represents an effective intervention against diabetic MI/RI (37). Collectively, these findings indicate that silencing AdipoR2 partially reversed the antioxidant and antiapoptotic effects of propofol postconditioning on $\mathrm{MI} / \mathrm{RI}$.

APN possesses the ability to restore STAT3 activation and reduce apoptosis, thus AdipoR2 can function as a mediator to prevent diabetic MI/RI through STAT3 (22). Moreover, the present study demonstrated that propofol postconditioning significantly upregulated STAT3 phosphorylation levels, whereas miR-200c-3p overexpression or AdipoR2 downregulation of AdiopR2 brought about the opposite effect. A recent study documented significant downregulation of STAT3 in I/R injury, which may serve a role in myocardial protection (38). Propofol was also previously shown to inhibit cardiomyocyte apoptosis in MI/RI by repressing the STAT3 pathway (39). Collectively, the results of the present study suggested that propofol postconditioning might have activated the STAT3 pathway and alleviated diabetic MI/RI via the miR-200c-3p/AdipoR2 axis.

In conclusion, results obtained in the present study indicated that propofol postconditioning could protect against MI/RI by inhibiting miR-200c-3p and activating the AdipoR2/STAT3 pathway. However, the protective effect of propofol postconditioning cannot be realized by a single and simple molecular mechanism. Whether propofol postconditioning can exert an effect by regulating other miRNAs is largely unknown. For example, miR-29b has been shown to protect against MI/RI (40). Furthermore, whether the protective effect of propofol postconditioning depends on miR-29b or other miRNAs remains to be studied. The regulation mechanism of propofol postconditioning on AdipoR2/STAT3 axis and whether miR-200c-3p could be a target of MI/RI in diabetes remains to be elucidated. The anti-oxidative and anti-apoptotic properties of propofol postconditioning could be attributed to complex processes of multiple pathways and receptors and the AdipoR2/STAT3 axis is only one of these mechanisms. The protective effect of propofol on MI/RI is not dose-dependent and the mechanism remains unclear. It may be possible that the internal lipid components of propofol can reduce the antioxidant effect of propofol during metabolism, however, this also needs further validation. The results of the present study have provided new ideas for the clinical treatment of diabetic MI/RI.

\section{Acknowledgements}

Not applicable.

\section{Funding}

No funding was received.

\section{Availability of data and materials}

The datasets used and/or analyzed during the current study are available from the corresponding author on reasonable request.

\section{Authors' contributions}

LH designed the study. LD, SY, XH and QR carried out the experiments and wrote the manuscript. LH and QR were 
responsible for reviewing and editing the manuscript. $\mathrm{LH}$ and LD confirm the authenticity of all the raw data. All authors read and approved the final manuscript.

\section{Ethics approval and consent to participate}

All procedures were authorized by the Academic Ethics Committee of The Affiliated People's Hospital of Ningbo University (Approval number: 2019-010). Experiments was carried out in strict accordance with the Guidelines for the Management and Use of Laboratory Animals issued by the Laboratory Association of China.

\section{Patient consent for publication}

Not applicable.

\section{Competing interests}

The authors declare that they have no competing interests.

\section{References}

1. Kerner W and Bruckel J; German Diabetes Association: Definition, classification and diagnosis of diabetes mellitus. Exp Clin Endocrinol Diabetes 122: 384-386, 2014.

2. Yu SY, Dong B, Fang ZF, Hu XQ, Tang L and Zhou SH: Knockdown of lncRNA AK139328 alleviates myocardial Ischaemia/reperfusion injury in diabetic mice via modulating miR-204-3p and inhibiting autophagy. J Cell Mol Med 22: 4886-4898, 2018.

3. Tao A, Xu X, Kvietys P, Kao R, Martin C and Rui T: Experimental diabetes mellitus exacerbates ischemia/reperfusion-induced myocardial injury by promoting mitochondrial fission: Role of down-regulation of myocardial Sirt1 and subsequent Akt/Drp1 interaction. Int J Biochem Cell Biol 105: 94-103, 2018.

4. Li J, Zhao Y, Zhou N, Li L and Li K: Dexmedetomidine attenuates myocardial ischemia-reperfusion injury in diabetes mellitus by inhibiting endoplasmic reticulum stress. J Diabetes Res 2019: 7869318, 2019.

5. Eltzschig HK and Eckle T: Ischemia and reperfusion-from mechanism to translation. Nat Med 17: 1391-1401, 2011.

6. Xu Y, Pan S, Jiang W, Xue F and Zhu X: Effects of propofol on the development of cancer in humans. Cell Prolif 53: e12867, 2020.

7. Yan HJ, Qi GQ and Ma Y: Effect of propofol on myocardial ischemia-reperfusion injury through MAPK/ERK pathway. Eur Rev Med Pharmacol Sci 23: 11051-11061, 2019.

8. Facey J, Young L and Nwokocha C: Relaxation responses of ketamine and propofol to vasoactive agents in Streptozotocin-induced diabetic rats. Niger J Physiol Sci 35: 33-39, 2020.

9. Deng F, Wang S, Zhang L, Xie X, Cai S, Li H, Xie GL, Miao HL, Yang C, Liu X and Xia Z: Propofol through upregulating Caveolin-3 attenuates Post-hypoxic mitochondrial damage and cell death in $\mathrm{H} 9 \mathrm{C} 2$ cardiomyocytes during hyperglycemia. Cell Physiol Biochem 44: 279-292, 2017.

10. Lin C, Sui H, Gu J, Yang X, Deng L, Li W, Ding W, Li D and Yang Y: Effect and mechanism of propofol on myocardial ischemia reperfusion injury in type 2 diabetic rats. Microvasc Res 90: 162-168, 2013.

11. Hao W, Zhao ZH, Meng QT, Tie ME, Lei SQ and Xia ZY: Propofol protects against hepatic ischemia/reperfusion injury via miR-133a-5p regulating the expression of MAPK6. Cell Biol Int 41: 495-504, 2017.

12. Lu TX and Rothenberg ME: MicroRNA. J Allergy Clin Immunol 141: 1202-1207, 2018.

13. Zhen W, Hui D, Wenying S and Yulong S: MicroRNA-20b-5p regulates propofol-preconditioning-induced inhibition of autophagy in hypoxia-and-reoxygenation-stimulated endothelial cells. J Biosci 45: 35, 2020.
14. Dehaini H, Awada H, El-Yazbi A, Zouein FA, Issa K, Eid AA, Ibrahim M, Badran A, Baydoun E, Pintus G and Eid AH: MicroRNAs as potential pharmaco-targets in ischemia-reperfusion injury compounded by diabetes. Cells 8: 152, 2019.

15. Climent M, Viggiani G, Chen YW, Coulis G and Castaldi A: MicroRNA and ROS crosstalk in cardiac and pulmonary diseases. Int J Mol Sci 21: 4370, 2020.

16. Zhao B, Gao WW, Liu YJ, Jiang M, Liu L, Yuan Q, Hou JB and Xia ZY: The role of glycogen synthase kinase 3 beta in brain injury induced by myocardial ischemia/reperfusion injury in a rat model of diabetes mellitus. Neural Regen Res 12: 1632-1639, 2017.

17. Li H, Yao W, Liu Z, Xu A, Huang Y, Ma XL, Irwin MG and $\mathrm{Xia} Z$ : Hyperglycemia abrogates ischemic postconditioning cardioprotection by impairing AdipoR1/Caveolin-3/STAT3 signaling in diabetic rats. Diabetes 65: 942-955, 2016.

18. Wang HY, Wang GL, Yu YH and Wang Y: The role of phosphoinositide-3-kinase/Akt pathway in propofol-induced postconditioning against focal cerebral ischemia-reperfusion injury in rats. Brain Res 1297: 177-184, 2009.

19. Zhu HJ, Wang DG, Yan J and Xu J: Up-regulation of microRNA-135a protects against myocardial ischemia/reperfusion injury by decreasing TXNIP expression in diabetic mice. Am J Transl Res 7: 2661-2671, 2015.

20. Agarwal V, Bell GW, Nam JW and Bartel DP: Predicting effective microRNA target sites in mammalian mRNAs. Elife 4: $\mathrm{e} 05005,2015$

21. Livak KJ and Schmittgen TD: Analysis of relative gene expression data using real-time quantitative PCR and the 2(-Delta C(T)) method. Methods 25: 402-408, 2001.

22. Wang T, Mao X, Li H, Qiao S, Xu A, Wang J, Lei S, Liu Z, Ng KF, Wong GT, et al: N-Acetylcysteine and allopurinol up-regulated the Jak/STAT3 and PI3K/Akt pathways via adiponectin and attenuated myocardial postischemic injury in diabetes. Free Radic Biol Med 63: 291-303, 2013.

23. Wang T, Qiao S, Lei S, Liu Y, Ng KF, Xu A, Lam KS, Irwin MG and Xia Z: N-acetylcysteine and allopurinol synergistically enhance cardiac adiponectin content and reduce myocardial reperfusion injury in diabetic rats. PLoS One 6: e23967, 2011.

24. Zhan L, Zhang Y, Su W, Zhang Q, Chen R, Zhao B, Li W, Xue R, $\mathrm{Xia} Z$ and Lei $\mathrm{S}$ : The roles of autophagy in acute lung injury induced by myocardial ischemia reperfusion in diabetic rats. J Diabetes Res 2018: 5047526, 2018

25. Li YM, Sun JG, Hu LH, Ma XC, Zhou G and Huang XZ: Propofol-mediated cardioprotection dependent of microRNA-451/HMGB1 against myocardial ischemia-reperfusion injury. J Cell Physiol 234: 23289-23301, 2019.

26. Wang Y, Che J, Zhao H, Tang J and Shi G: Platycodin D inhibits oxidative stress and apoptosis in $\mathrm{H} 9 \mathrm{c} 2$ cardiomyocytes following hypoxia/reoxygenation injury. Biochem Biophys Res Commun 503: 3219-3224, 2018.

27. Rahimi R, Karimi J, Khodadadi I, Tayebinia H, Kheiripour N, Hashemnia M and Goli F: Silymarin ameliorates expression of urotensin II (U-II) and its receptor (UTR) and attenuates toxic oxidative stress in the heart of rats with type 2 diabetes. Biomed Pharmacother 101: 244-250, 2018.

28. Hu C, Zhang X, Wei W, Zhang N, Wu H, Ma Z, Li L, Deng W and Tang Q: Matrine attenuates oxidative stress and cardiomyocyte apoptosis in doxorubicin-induced cardiotoxicity via maintaining AMPK $\alpha /$ UCP2 pathway. Acta Pharm Sin B 9: 690-701, 2019.

29. Chen Y and Li Z: Protective effects of propofol on rats with cerebral ischemia-reperfusion injury via the PI3K/Akt Pathway. J Mol Neurosci 71: 810-820, 2021.

30. Li H, Zhang X, Tan J, Sun L, Xu LH, Jiang YG, Lou JS, Shi XY and Mi WD: Propofol postconditioning protects H9c2 cells from hypoxia/reoxygenation injury by inducing autophagy via the SAPK/JNK pathway. Mol Med Rep 17: 4573-4580, 2018.

31. Tang M, Liu P, Li X, Wang JW, Zhu XC and He FP: Protective action of B1R antagonist against cerebral ischemia-reperfusion injury through suppressing miR-200c expression of Microglia-derived microvesicles. Neurol Res 39: 612-620, 2017.

32. Wu Y, Gu C and Huang X: Sevoflurane protects against hepatic ischemia/reperfusion injury by modulating microRNA-200c regulation in mice. Biomed Pharmacother 84: 1126-1136, 2016.

33. Shi D, Guo L, Sun X, Shang M, Meng D, Zhou X, Liu X, Zhao Y and Li J: UTMD inhibit EMT of breast cancer through the ROS/miR-200c/ZEB1 axis. Sci Rep 10: 6657, 2020.

34. Nunomura A and Perry G: RNA and oxidative stress in Alzheimer's disease: Focus on microRNAs. Oxid Med Cell Longev 2020: 2638130, 2020. 
35. Zhang Y, Zhao J, Li R, Lau WB, Yuan YX, Liang B, Li R, Gao EH, Koch WJ, Ma XL and Wang YJ: AdipoRon, the first orally active adiponectin receptor activator, attenuates postischemic myocardial apoptosis through both AMPK-mediated and AMPK-independent signalings. Am J Physiol Endocrinol Metab 309: E275-E282, 2015.

36. Li D, Song LL, Wang J, Meng C and Cui XG: Adiponectin protects against lung ischemia-reperfusion injury in rats with type 2 diabetes mellitus. Mol Med Rep 17: 7191-7201, 2018.

37. Wang Y, Liang B, Lau WB, Du Y, Guo R, Yan Z, Gan L, Yan W, Zhao J, Gao E, et al: Restoring diabetes-induced autophagic flux arrest in ischemic/reperfused heart by ADIPOR (adiponectin receptor) activation involves both AMPK-dependent and AMPK-independent signaling. Autophagy 13: 1855-1869, 2017.

38. Zhang Y, Zheng LM, Wang CX, Gu JM and Xue S: SENP3 protects $\mathrm{H} 9 \mathrm{C} 2$ cells from apoptosis triggered by H/R via STAT3 pathway. Eur Rev Med Pharmacol Sci 22: 2778-2786, 2018.
39. Chen X, Wang Y, Xiao ZY, Hou DN, Li DB and Zhang XP: Effect of propofol on myocardial ischemia/reperfusion injury in rats through JAK/STAT signaling pathway. Eur Rev Med Pharmacol Sci 23: 6330-6338, 2019.

40. Li K, Zhou P, Li S, Zheng S and Wang D: MicroRNA-29b reduces myocardial ischemia-reperfusion injury in rats via down-regulating PTEN and activating the Akt/eNOS signaling pathway. J Thromb Thrombolysis 53: 123-135, 2021.

This work is licensed under a Creative Commons Attribution-NonCommercial-NoDerivatives 4.0 International (CC BY-NC-ND 4.0) License. 